\title{
Undergraduate optical experiments using simple liquid crystal displays
}

Silvia Ledesma, Homero Lozza, Matias Pomata

Silvia A. Ledesma, Homero Lozza, Matias Pomata, "Undergraduate optical experiments using simple liquid crystal displays," Proc. SPIE 3831, Sixth International Conference on Education and Training in Optics and Photonics, (16 June 2000); doi: 10.1117/12.388738

Event: Education and Training in Optics and Photonics (ETOP'99), 1999, Cancun, Mexico 


\title{
Undergraduate optical experiments using simple liquid crystal displays
}

\author{
Silvia Ledesma*, Homero Lozza, Matías Pomata
}

\author{
Laboratorio de Procesado de Imágenes, Departamento de Física, Facultad de Ciencias Exactas y \\ Naturales, Universidad de Buenos Aires, Buenos Aires, Argentina.
}

\begin{abstract}
We propose here the use of few pixel liquid crystal displays (LCD) to be used in optics experiments. Commercial LCD displays used in electronic sensors have a simple electronic, can be addressed by conventional wavegenerators and are, in addition, very cheap. These devices are governed by the same basic principles as the recently developed high-resolution crystal displays. The physical models that explain the behaviour of the LCD are very interesting from an educational point of view. The predictions arising from different models can be compared by performing simple photometric measurements. By means of interferometric architectures it is possible to measure the phase modulation introduced in the interference pattern when different voltages are applied to the LCD. These devices have the ability of provide a controlled phase modulation, a feature that renders them very useful devices for phase shift interferometers. The experiences presented here can be performed in an undergraduate laboratory with a basic optical equipment.
\end{abstract}

Keywords: optical education, liquid crystal panels

\section{INTRODUCTION}

Liquid crystal panels in nematic phase are commonly used for displays in electronic watches, electronic games, calculators, computers, etc. Their application to real time optical image processing has begun recently ${ }^{1,2,3,7}$.

A liquid crystal display is typically composed of multiple layers. The first one is a glass plate covered by a transparent film working as an electrode. The area where each pair of electrodes modifies the refractive index of the crystal is called pixel. On top of the electrodes there is a grooved polymer layer. The axes of the crystal molecules align themselves mainly along the direction of the grooves. The same elements follow the crystal in an inverse order, namely, polymer, electrode and glass. The grooves of the second polymer layer are rotated an angle $\alpha$ with respect to the grooves of the first. The crystal molecules between the glass plates are therefore disposed in thin layers to form a helical structure, in such a way that the axes of the ones in the first layer are twisted an angle $\alpha$ with respect to the axes of the molecules of the last layer.

The most commonly used configuration is the one known as "twisted nematic" where $\alpha=90^{\circ}$. By adding a pair of polarizers with their transmission axes parallel to the optical axes at the sides of the display and applying a small voltage, it is possible to selectively darken some zones in the display to create graphics or alphanumeric characters.

The beam incident on the pixel is polarized in the direction of the director molecules of the first layer. In an adiabatic regime the incident light rotates its polarization following the twist angle of the molecules. When the light reaches the last layer the polarization has been rotated $90^{\circ}$ and is completely transmitted through the second polarizer (if the attenuation of this latter is not taken into account).

When an electric field is applied the molecules close to the electrodes remain in their place, but the others rotate to align their axes with the field direction. The helix structure is broken and the light passes through the crystal preserving the

\footnotetext{
* Correspondence: Pabellón I, Ciudad Universitaria (1428), Capital Federal, Argentina.

e-mail: ledesma@df.uba.ar,
} 
polarization of the incident light. Provided that the second polarizer is orthogonal to the first one, the light is completely absorbed.

We present here a study of a commercial liquid crystal display of $3 \frac{1}{2}$ digits, made by AND, model FE0203 [Fig. 1] usually used to display numbers using seven segments. This display can be bought in any electronic shop, is very cheap and can be addressed by a simple wave function generator. Furthermore, the pixel size is large enough to allow detection only on the active zones of the liquid crystal. We have characterised this liquid crystal panel using two alternative models to describe its response to the applied voltage. We have measured the liquid crystal parameters and the polarization, intensity and phase modulation as a function of the voltage applied to the electrodes. A photograph of the used LCD is shown in Figure 1.

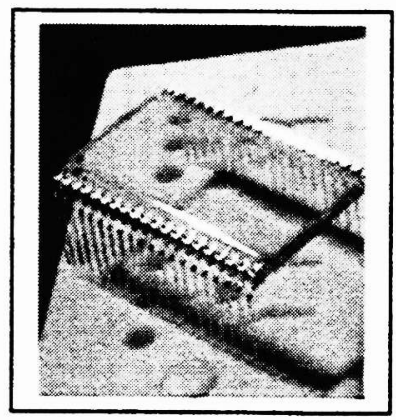

Figure 1: Liquid crystal panel AND, model FE0203

\section{MODELLING THE CRYSTAL RESPONSE}

\subsection{Adiabatic response}

Let us consider a coordinate system with the $\mathrm{z}$ axis parallel to the propagation direction of the electromagnetic field. Each layer of the liquid crystal will be considered as a retarder plate. Let us choose the $\mathrm{x}$ direction as parallel to the slow axis $\mathrm{S}$ of the first plate and $y$ direction parallel to the fast one, F. In Figure 2 we show the set of layers.

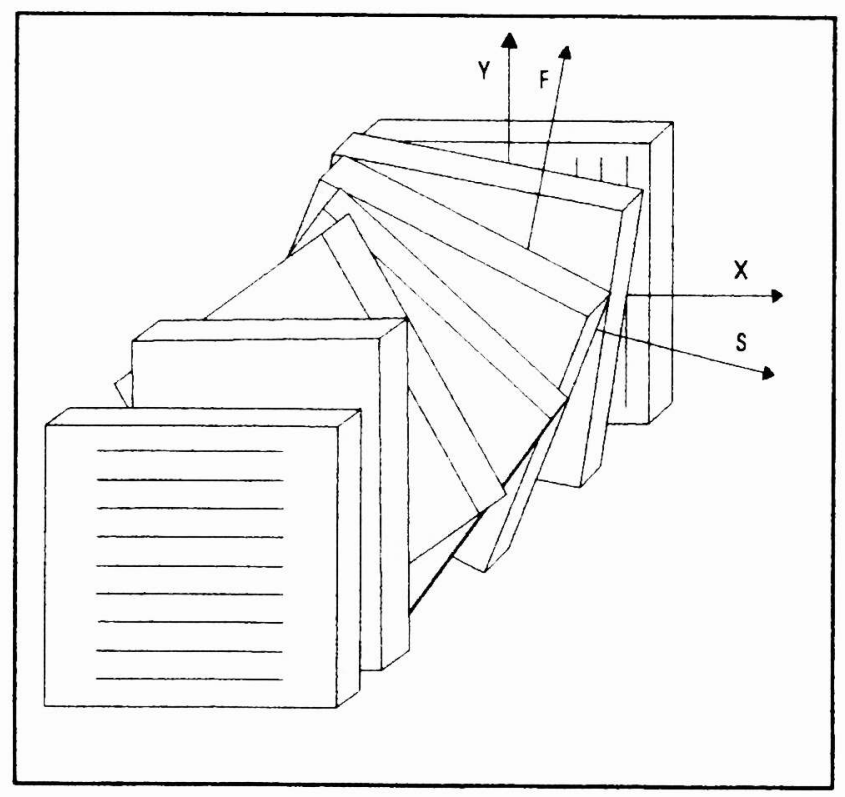

Figure 2: Model for a twisted nematic liquid crystal 
It will be assumed that the twist angle of each plate $\Psi(\mathrm{z})$ has a linear dependence on the distance to the first plate

$$
\psi(\mathrm{z})=\mathrm{c} . \mathrm{z}
$$

where $\mathrm{z}$ is the distance in the propagation direction and $\mathrm{c}$ is a constant. Therefore,

Let $\beta=\Gamma / 2$ be the optical path difference that would result if the optical axes of all the retarder plates were parallel.

$$
\beta=\pi\left(\mathrm{n}_{\mathrm{e}}-\mathrm{n}_{0}\right) \mathrm{d} / \lambda
$$

where $d$ is the distance between the inner faces of the glasses and $\lambda$ is the wavelength of the incident light. The total twist angle is then given by

$$
\alpha=\mathrm{c} . \mathrm{d}
$$

We will consider the liquid crystal structure as composed by $\mathrm{N}$ retarder plates of the same thickness. The optical path difference through of each plate is $\beta / \mathrm{N}$ and the principal axes form angles $\rho, 2 \rho, \ldots, \mathrm{N} \rho$, with respect to the first one, with $\rho=\alpha / \mathrm{N}$.

It can be demonstrated that in the limit of $\mathrm{N}$ tending to infinite, the transmission matrix $M_{l}$ is given by

$$
M_{1}=e^{-i \phi} R(\alpha)\left[\begin{array}{cc}
\cos (\gamma)-i \frac{\beta \cdot \sin (\gamma)}{\gamma} & -\frac{\alpha \cdot \sin (\gamma)}{\gamma} \\
\frac{\alpha \cdot \sin (\gamma)}{\gamma} & \cos (\gamma)+i \frac{\beta \cdot \sin (\gamma)}{\gamma}
\end{array}\right]
$$

where $\gamma=\sqrt{\alpha^{2}+\beta^{2}}$ and $\quad R(\alpha)$ is the rotation matrix of angle $\alpha$.

The total transmission of the crystal depends on the ordinary and extraordinary refractive indexes, according to Eq. (2). However, since the optical axis rotates with the applied voltage, the refractive indexes are $n_{0}$ and $n_{e}(\theta)$, where $\theta$ is the angle between the optical axis and the direction of the electric field, which depends on the applied voltage. In our case the direction of the light propagation and the direction of the electric field are parallel to $z$, and therefore it follows that

$$
\frac{1}{n_{e}^{2}(\theta)}=\frac{\cos ^{2}(\theta)}{n_{e}^{2}}+\frac{\sin ^{2}(\theta)}{n_{0}^{2}}
$$

The relationship between $\theta$ and the applied voltage is given by

$$
\theta=\left[\begin{array}{cc}
0 & V<V_{c} \\
\frac{\pi}{2}-2 \operatorname{ArcTan}\left\{e^{-\frac{\left(V-V_{c}\right)}{V_{0}}}\right\} & V \geq V_{c}
\end{array}\right.
$$

where $V_{c}$ is a threshold voltage below which the molecules do not rotate and $V_{0}$ is defined as a voltage that produces by an angle $49.6^{\circ}$ between the molecular director axis and the $\mathrm{z}$ direction. 


\subsection{Semi-adiabatic response}

As mentioned in the first section, the molecules closest to the glasses remain with the same orientation even in the case of intense applied voltages. A more realistic model considers the existence of two fixed retarder plates, one located at the beginning, and another one at the end, of the set of retarder plates proposed in the first model ${ }^{[8]}$. In this way it is possible to compensate the difference between the action of the field close and far from the glasses.

Let us call $\mathrm{W}_{1}$ and $\mathrm{W}_{2}$ the Jones matrixes for the above mentioned plates

$$
W_{1}=\left[\begin{array}{cc}
e^{-i \delta} & 0 \\
0 & 1
\end{array}\right], W_{2}=\left[\begin{array}{cc}
1 & 0 \\
0 & e^{-i \delta}
\end{array}\right]
$$

where $\delta$ is the optical path difference. The total transmission matrix will be

$$
M_{2}=W_{2} \cdot M_{1} \cdot W_{1}
$$

\section{EXPERIMENTS}

\subsection{Crystal parameters}

There are different parameters that should be determined for each particular crystal: the twist angle of the molecules, $\alpha$, the extraordinary refractive index $n_{e}$ and the ordinary refractive index $n_{0}$. We have determined these parameters with the use of the first model.

Generally, the technical specifications given by the manufacturers of liquid crystals do not include the data necessary for this type of optical experiments, since they are mainly oriented to electronic circuit designers. Furthermore, it is possible to find many displays with an integrated control logic that do not allow exploration the total work range, due to the limitations imposed by the electronic circuits.

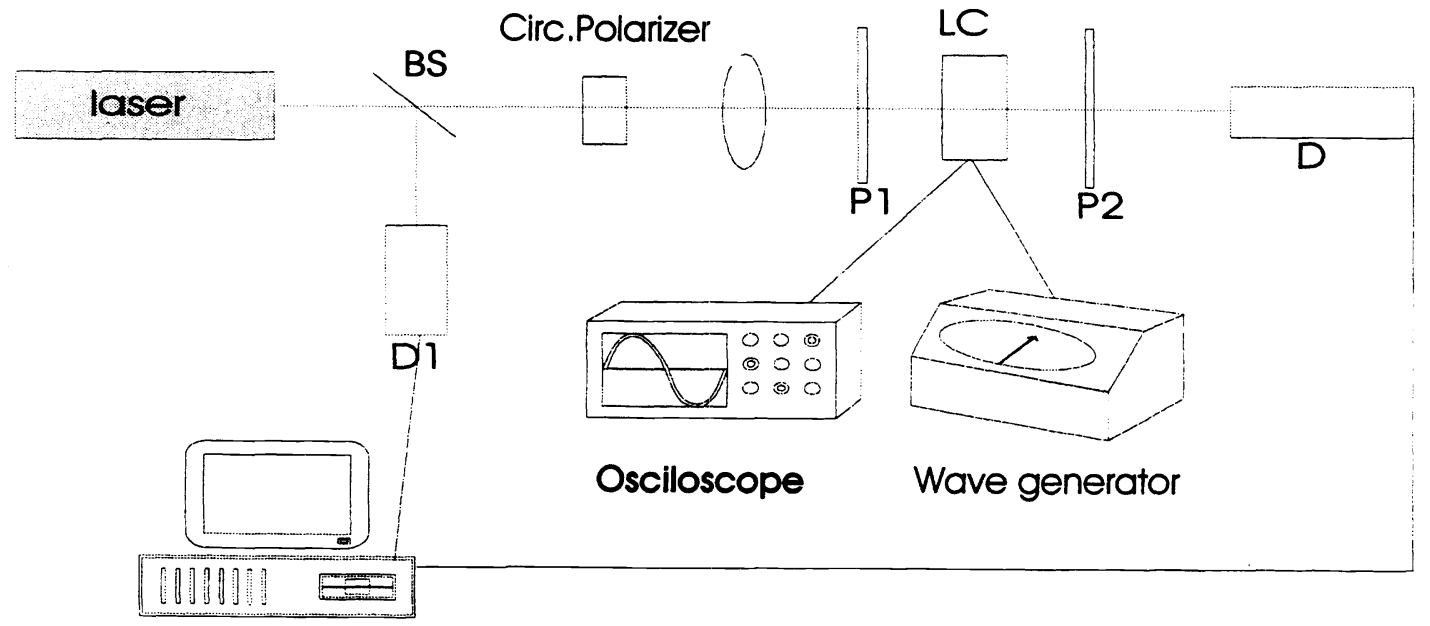

PC

Figure 3. Experimental setup: BS is a beam splitter; D1 and D2 are detectors; P1 and P2 are polarizers. The PC has a multipurpose lab interface with three channels. 
To measure the transmission corresponding to different polarizations and different applied voltages we have employed an experimental arrangement sketched in Figure 3.

A polarized He-Ne laser of wavelength $632.8 \mathrm{~nm}$ impinges on a beam splitter. One beam is monitored with the detector D1, to control the intensity laser fluctuations. A circular polarizer is used to produce comparable intensities when the crystal input polarization is modified.

We have focalised the laser beam to ensure utilisation of the active zone. The crystal was placed between two polarizers, $\mathrm{P} 1$ and $\mathrm{P} 2$. We have measured the transmitted intensity using a second detector, $\mathrm{D}_{2}$.

The usual method to control one pixel is to use a pulsed continuous signal with amplitude $\mathrm{V}$ applied to one of the electrodes called the 'backplane' (common to all pixels). Then an identical signal is applied to the electrodes corresponding to the segments of those pixels that will be deactivated or an identical signal dephased in $180^{\circ}$ is applied to the pixels that will be activated. Therefore, since the voltage over each pixel is the potential difference between the electrodes, there is no applied voltage on the deactivated pixels, and on the activated pixels there is an alternating square signal of zero DC and of $2 \mathrm{~V}$ peak to peak amplitude.

Since there is an alternating applied voltage, the period of the signal should be much shorter than the viscoelastic response time of the liquid crystal. In such case the optical response is determined by the root mean square of the voltage. By means of a wave function generator we introduced a square signal of $40 \mathrm{~Hz}$ with zero mean value and with an amplitude of $10 \mathrm{Vpp}$ between the backplane and the terminal of the pixel that we want to activate, and we scanned all the voltages between 0 and $10 \mathrm{Vpp}$.

To automatize this process we have selected the AM modulator of the generator. Finally we have worked with a carrying signal of $40 \mathrm{~Hz}$ and $10 \mathrm{Vpp}$ and with a triangular modulating signal of $30 \mathrm{mHz}$ and $100 \%$ of modulation depth. For the triangular signal we had a linear scanning and a modulating frequency of $30 \mathrm{mHz}$, which was 100 smaller than the excitation signal selected to work in quasi static conditions. The excitation and modulating signals are shown in Figure 4.

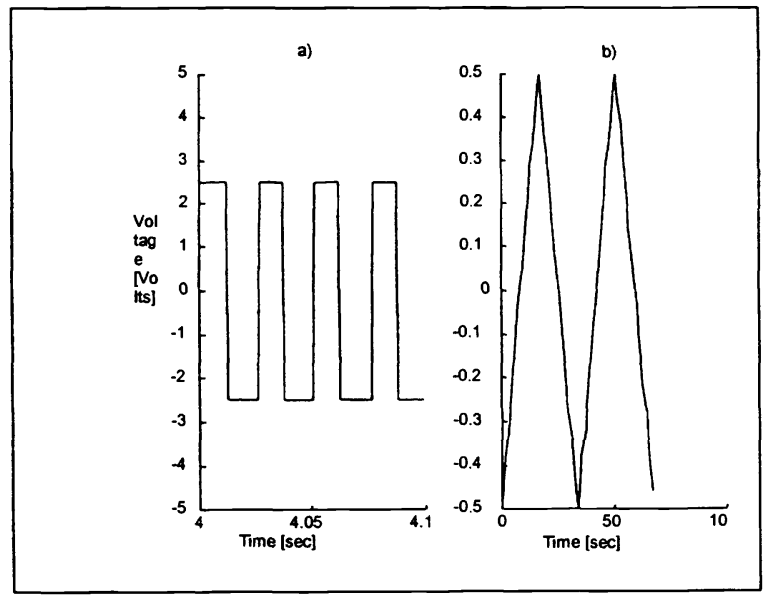

Figure 4: a) Excitation signal, b) Modulating signal

Neither of the theoretical models that we want to compare take into account the absorption in the medium or the internal reflections. We have normalised the measured component to the total output intensity. 


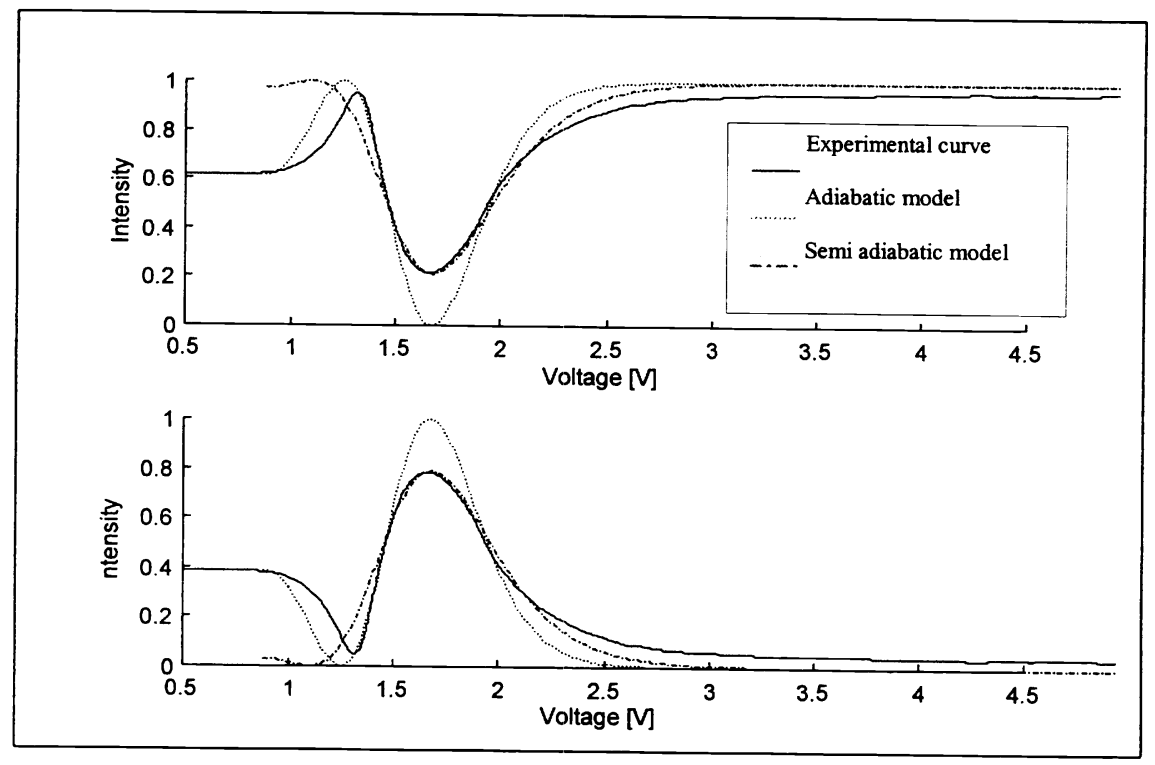

Figure 5: Transmited intensity for $\psi_{1}=45^{\circ}$, a) $\psi_{2}=45^{\circ}$ b) $\psi_{2}=-45^{\circ}$

We have obtained the parameters of the liquid crystal from the first model, with no applied voltage. This choice was based on the fact that for zero voltage the molecules do not rotate on the direction of the electric field, so the influence of those molecules that composed the fixed retarder plates against the glasses in the second model is negligible.

To obtain the phase $\delta$ corresponding to the second model, we have adjusted the extremes of the experimental and the theoretical curves with $\psi 1=\psi 2=45^{\circ}$ showed in Figure 5. Provided that $\lambda$, ne and no are known we can find the thickness of the plates which are fixed to the sides according to this model. From the obtained value of $\delta$ we have found that the thickness of each fixed plate is about $15 \%$ of the total crystal thickness. Finally the thickness of the plates must be subtracted of the total thickness $d$ to obtain the region where the crystal is sensible to the applied voltage.

\subsection{Commutation}

There are two configurations that yield maximum and minimum values for the extremes of the transmission versus voltage curves. For the case $\psi 1=\psi 2=0^{\circ}$ and zero voltage, the transmission is very low and for a voltage close to $5 \mathrm{~V}$ the transmission approaches one. On the contrary, for $\psi 1=0^{\circ}$ and $\psi 2=90^{\circ}$ the transmission tends to unity when no voltage is applied, and when the voltage is approximately $5 \mathrm{~V}$ the transmission is almost zero. Using these results it is possible to pulse a light beam that impinges on one pixel of the LCD by applying a commuting voltage, between $0 \mathrm{~V}$ and $5 \mathrm{~V}$.

We have found that the used LCD can work as a key at frequencies smaller than $1 \mathrm{~Hz}$. For frequencies up to $1 \mathrm{~Hz}$ the transient is not observed but when this value is exceeded the transient time is comparable to the commutation time. In Figure 6 we show the intensity curves for an excitation signal of $40 \mathrm{~Hz}$ modulated with square signals of $0.1 \mathrm{~Hz}, 0.3 \mathrm{~Hz}$ and $1 \mathrm{~Hz}$. 

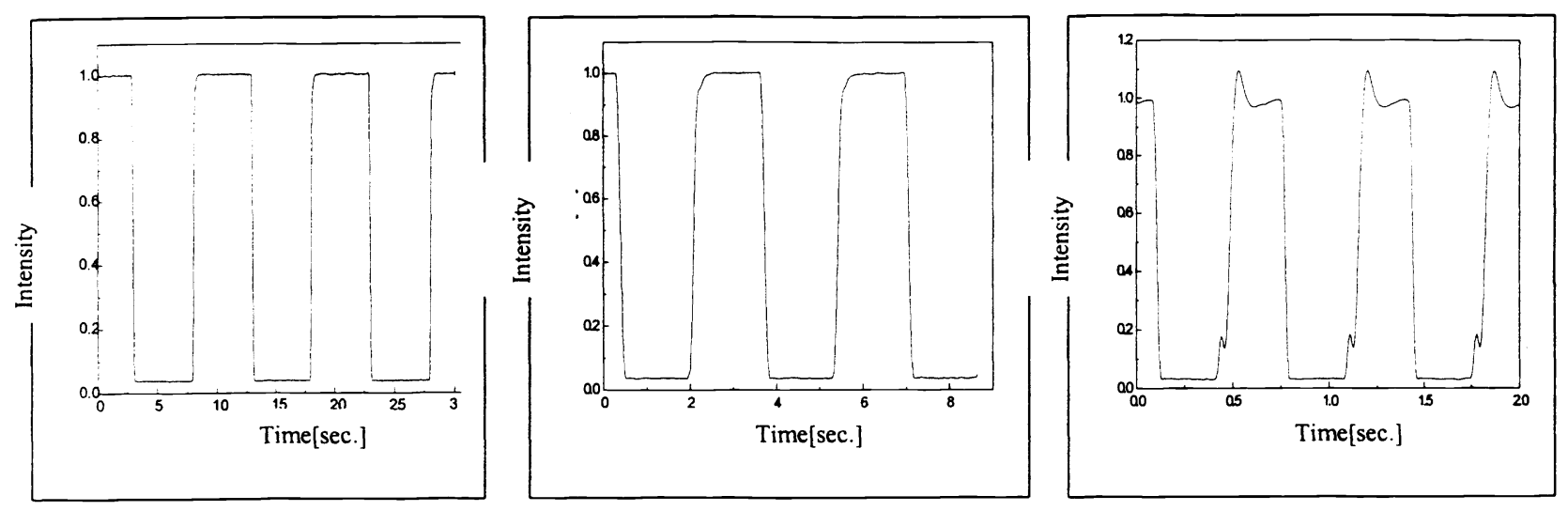

Figure 6: a) Commutation at $100 \mathrm{mHz}$, b) Conmutation at $300 \mathrm{mHz}$, c) Commutation at $1 \mathrm{~Hz}$

\subsection{Phase mode}

For optical signal processing as well as for interferometric applications it is interesting to have configurations where the amplitude transmission and the phase transmission are decoupled. By using two linear polarizers, one at the entrance and another one at the exit of the LCD, one can seek the orientation for which the phase varies from 0 to $2 \pi$ and the amplitude has minimum modulation ${ }^{[4]}$. Recently ${ }^{[9]}$ a new set-up for this goal has been proposed, that makes use of the elliptic eigenstates of the liquid crystal.

\subsubsection{Phase mode applications}

In phase shift interferometric techniques it is necessary to vary the phase in one arm of the interferometer in a controlled way. This type of interferometry is based on the production of 3 or 4 interference patterns with a phase shift between 0 and $2 \pi$. On the other hand, in optical pattern recognition liquid crystal television panels are used to display both the filter function and the scene. For architectures based on a Fourier transform procedure, a high efficiency is wanted to get high illumination. This is achieved by modulating the phase of the liquid crystal with the signal that carries the information while keeping the amplitude transmission as close to the unity as possible. In general it is very difficult to obtain decoupled amplitude and phase transmissions.

We propose here an experimental setup to be used with a Michelson type interferometer, which uses only one linear polarizer at the input of the liquid crystal to obtain a wide range of phase modulation. In this case no amplitude modulation is predicted by theory. We present theoretical and experimental curves of the phase modulation as a function of the applied voltage where a phase range from 0 to $2 \pi$ has been obtained.

\subsubsection{Experimental setup}

A scheme of the Michelson type interferometer we have used is shown in Figure 7. A linear polarized He-Ne laser of wavelength $632.8 \mathrm{~nm}$, expanded and collimated, was employed. The direction of the input polarization was selected to obtain high contrast and a minimum phase modulation. In one of the arms of the interferometer a LCD was placed. The laser beam illuminated the activated pixel and the surrounding region. The activated pixel was addressed with a conventional wave function generator 
A CCD connected to a PC registered the interference patterns produced by the superposition of the light passing through the two arms of the interferometer.

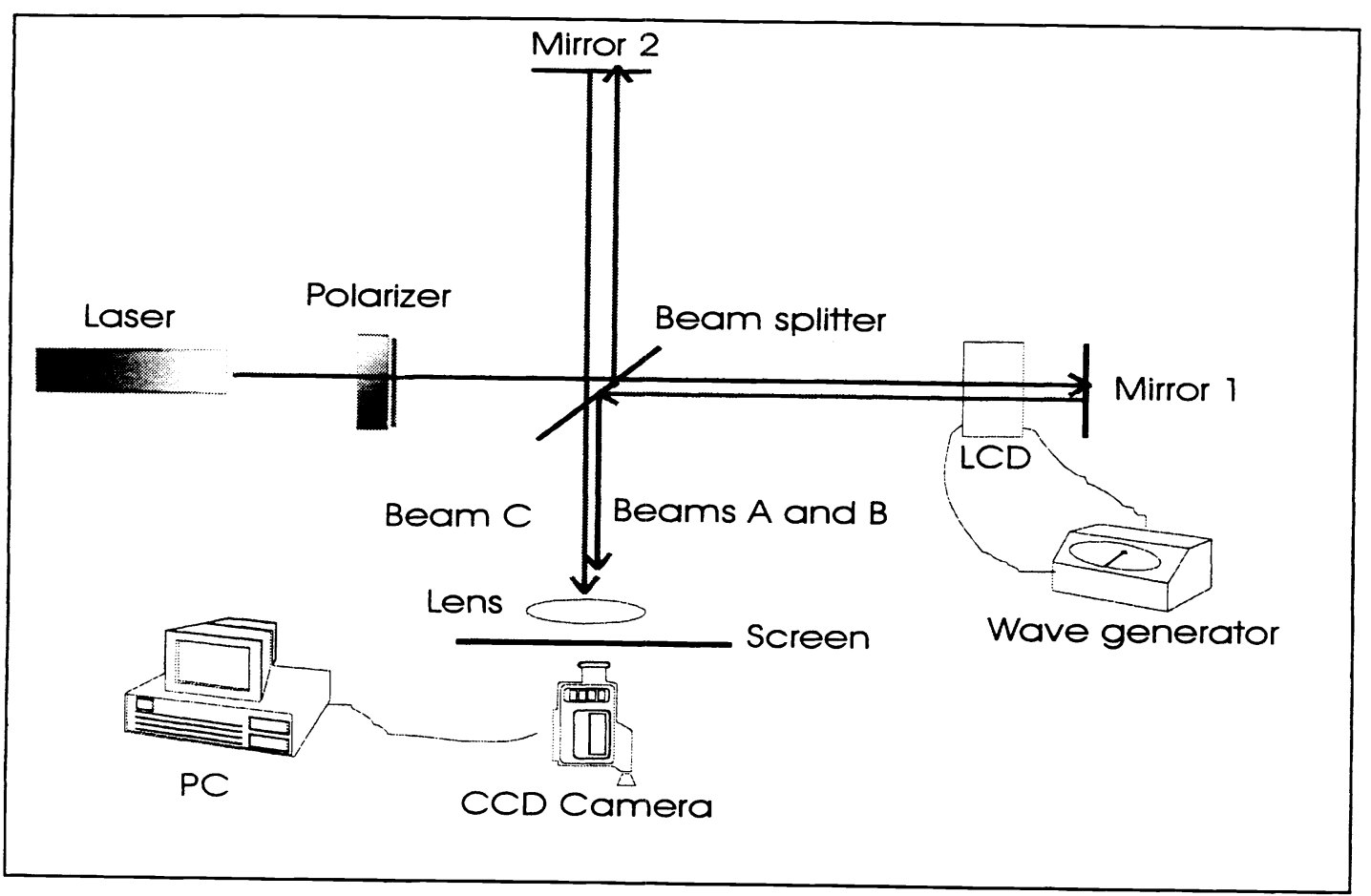

Figure 7: Michelson type interferometer for measuring the relative phase shift.

Since the incident light was linearly polarized, after passage through the liquid crystal two elliptically polarized light beams are obtained, as shown in Figure 8. The beam A impinges on the non activated zone of the liquid crystal and is reflected by the mirror. It then passes again through the non activated zone of the liquid crystal. We shall call $\vec{E}^{\prime}(V=0)$ the electric field vector in this beam. The beam B impinges on the non activated zone of the crystal but after reflection on the mirror it passes through the activated zone. Let us call $\vec{E}^{\prime}(V)$ the field in this beam. In this way, polarization, intensity and phase are constant for the A beam, but they vary with the applied voltage for the B beam.

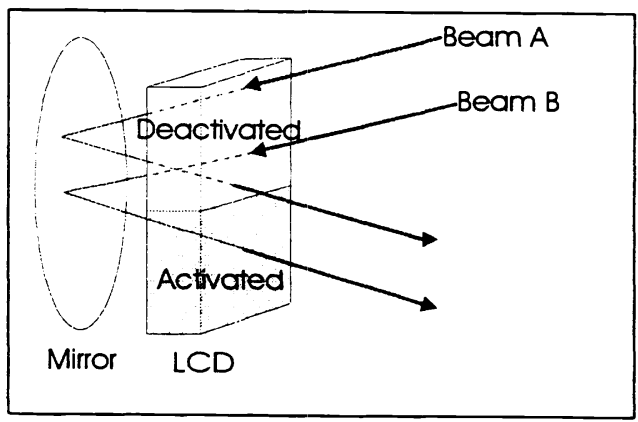

Figure 8: Scheme used in the model. 
The beams $\mathrm{A}$ and $\mathrm{B}$ will interfere with the beam $\mathrm{C}$ with electric field $\vec{E}_{c}$ that arrives from the other interferometer arm. Let us call $\vec{E}_{0}^{\prime}$ and $\vec{E}_{1}^{\prime}$ the projections of $\vec{E}^{\prime}(V)$ on two perpendicular directions. The resulting electric field will be

$$
\vec{E}_{\text {total }}^{\prime}=\vec{E}_{0}^{\prime}+\vec{E}_{1}^{\prime}+\vec{E}_{c}^{\prime}
$$

The intensity can be written as

$$
I=\vec{E}_{\text {total }}^{\prime} \cdot\left(\vec{E}_{\text {total }}^{\prime}\right)^{*}=E_{0}^{\prime 2}+E_{1}^{\prime 2}+E_{2}^{\prime 2}+2 \operatorname{Re}\left[\vec{E}_{0}^{\prime} \cdot \vec{E}_{1}^{\prime *}+\left(\vec{E}_{0}^{\prime}+\vec{E}_{1}^{\prime}\right) \cdot \vec{E}_{c}^{\prime *}\right]
$$

The term $\vec{E}_{0}^{\prime} \cdot \vec{E}_{1}^{\prime *}$ is identically zero because these vectors are orthogonal. Therefore the phase difference arises from the term

$$
\left(\vec{E}_{0}^{\prime}+\vec{E}_{1}^{\prime}\right) \cdot \vec{E}_{c}^{\prime *}=\vec{E}^{\prime} \cdot \vec{E}_{c}^{\prime *}=E^{\prime} \cdot E_{c}^{\prime} \cos (\varphi)
$$

The phase difference produced by the activated zone can be calculated as

$$
\varphi=\beta-\operatorname{ArcTan}\left(\frac{\operatorname{Im}\left[\vec{E}^{\prime}(V) \cdot \vec{E}_{c}^{\prime *}\right]}{\operatorname{Re}\left[\vec{E}^{\prime}(V) \cdot \vec{E}_{c}^{\prime}\right]}\right)+\beta_{0}-\operatorname{ArcTan}\left(\frac{\operatorname{Im}\left[\vec{E}^{\prime}(V=0) \cdot \vec{E}_{c}^{\prime}\right.}{\operatorname{Re}\left[\vec{E}^{\prime}(V=0) \cdot \vec{E}_{c}^{\prime}\right]}\right)
$$

We have observed that the input polarization corresponding to the maximum phase modulation and maximum contrast was $\psi=-\frac{\pi}{4}$, measured with respect to the director axis of the liquid crystal. We have evaluated the liquid crystal response for this incident polarization.

By controlling the mirror positioners we have selected the optimal fringe frequency for our experiment. When a voltage is applied, a phase difference between the activated and the non activated zone is introduced. This in turn produces a variation in the position of the fringes in each zone. In Figure 9 we show the measured and calculated phase differences to our configuration.

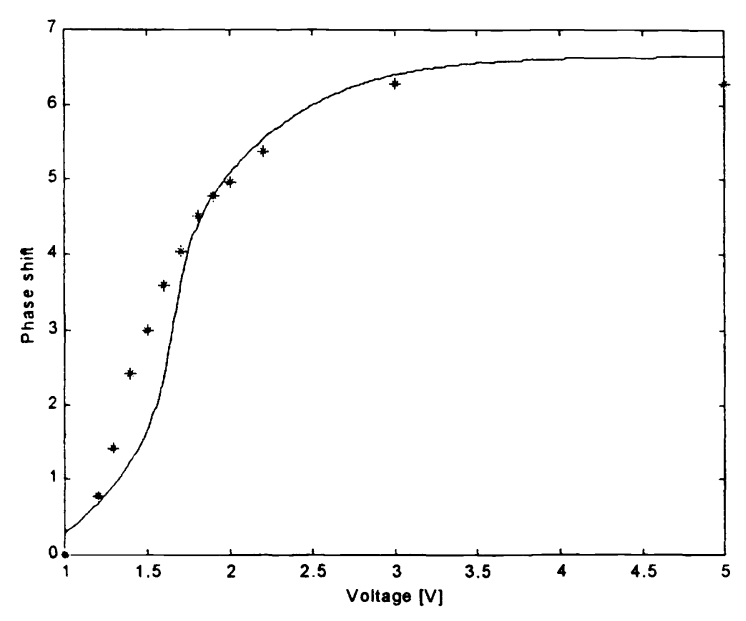

Figure 9: Phase shift vs. applied voltage. 
As we can observe from the figure a complete modulation from zero to $2 \pi$ has been obtained.

\section{SUMMARY}

We have used a standard commercial liquid crystal panel addressed by a wave function generator to explore the physical phenomena involved in its operation. The experiments proposed here can be performed in a basic undergraduate optics laboratory, and can be used to demonstrate basic concepts such as polarization, birefringence, phase modulation and interference in elementary optics courses. In advanced courses, by making use of the complete range of the panel, students can analyse and compare different physical models that describe the behaviour of the liquid crystal molecules.

\section{ACKNOWLEDGEMENTS}

S. Ledesma acknowledges the financial support from CONICET and UBA.

\section{REFERENCES}

1. Handbook of Liquid Cristal Research,P.J.Collins and J.S.Patel.Editors in chief. Oxford University Press.

2. I.M.Soriano. Tesis de Doctorado. Facultat de Ciéncies; Universitat Autónoma de Barcelona. "Diseño de sistemas multicanales en tiempo real para el reconocimiento de objetos policromáticos".

3. T.H.Barnes,T.Eiju,K.Matsuda,N.Ooyama, "Phase only modulation using a twisted nematic liquid cristal television". Applied Optics/Vol.28,N²2/15 November 1989.

4. K.Lu,B.Saleh, "Theory and design of a liquid cristal TV as an optical spatial phase modulator". Optical Engineering/Vol 29, $\mathrm{N}^{\circ} 3 /$ March 1990.

5. J.C.Kirsch,D.A.Gregory,M.W.Thie,B.K.Jones, "Modulation characteristics of the Epson liquid cristal television". Optical Engineering 31(5), 963-970(March 1992).

6. K.Ohkubo,J.Ohstubo, "Evaluation of LCTV as espatial light modulator". Optics Communications 102(1993) 116-124.

7. L.Goncalves Neto,D.Roberge,Y.Sheng, "Programmable optical phase-mostly holograms with coupled mode modulation liquid cristal television". Applied Optics/Vol. 34,N 11/10 April 1995.

8. J.A.Coy,M.Zaldarriega,D.F.Grosz,O.E.Martinez, "Characterization of a liquid cristal television as a programmable spatial light modulator". Optical Engineering/January 1996/Vol.35, $\mathrm{N}^{\circ} 1$.

9. J.A.Davis,I.Moreno,P.Tsai, "Polarization eigenstates for twisted-nematic liquid-cristal displays" Applied Optics/Vol.37,N5/10 February 1998. 